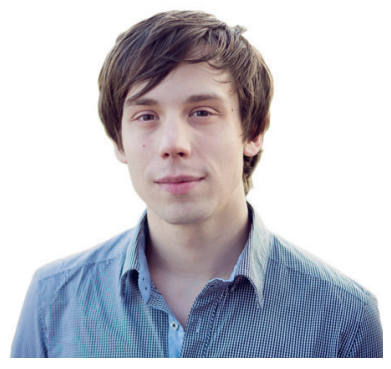

\title{
Atopie als Vorbote von Autoimmunerkrankungen - Nicht aus dem (zweiten) Blick verlieren
}

\section{David Brandt}

Universitäts Kinder- und Frauenzentrum, Klinik für Kinder- und Jugendmedizin, Bereich Bronchopneumologie/Allergologie, Dresden, Deutschland

Abstract aus Hassidim A, Merdler I, Chorin O, et al.: Atopic predilection among kawasaki disease patients: a cross-sectional study of 1,187,757 teenagers. Int Arch Allergy Immunol 2016;170:92-96.

\section{Keywords}

Kawasaki disease · Allergic rhinitis · Asthma · Angioedema ·

Urticaria

\begin{abstract}
Background: Kawasaki disease (KD) is an acute, systemic vasculitis in children, with an etiology that is not completely understood. It is assumed that the development of KD is mediated by an immunologic response. Several reports from East Asia have found a higher prevalence of atopic diseases among patients with KD, but a large-scale study of a non-Asian population regarding this correlation is still lacking. The purpose of this article was to achieve this goal.
\end{abstract}

Methods: We conducted a cross-sectional, large-scale study to estimate the correlation of KD with allergic diseases. The medical history of 1,187,757 Israeli teenagers (aged 16-20 years during the years 1998-2013) was retrieved. The study population was divid- ed into 3 groups according to a past history of noncomplicated and complicated KD and a control group. The prevalence of allergic diseases among these groups was further investigated.

Results: The prevalence of atopic diseases in the 3 study groups was presented (asthma in 11.4, 8.1 and 3.5\%, respectively; angioedema/urticaria in $7.1,0$ and $0.46 \%$, respectively; allergic rhinitis in 20, 12.1 and 6.7\%, respectively). In noncomplicated KD, a statistically significant link to asthma [odds ratio (OR) 2.4; $p=0.048$ ] and a borderline significant link to allergic rhinitis (OR 1.9; $p=0.06$ ) were found. In KD complicated with cardiac disease, statistically significant links were found for all the allergic conditions, asthma (OR 3.5; $p=0.003$ ), allergic rhinitis (OR 3.5; $p<0.001$ ) and angioedema/urticaria (OR 16.48; $p<0.001)$.

Conclusion: KD is associated with allergic diseases. This association increases with the severity of the disease.

(c) 2016 S. Karger AG, Basel

\section{KARGER}

(C) 2019 S.Karger GmbH, Freiburg 


\section{Transfer in die Praxis}

\section{Hintergrund}

Auf den ersten Blick haben atopische Erkrankungen und Autoimmunerkrankungen wenig gemeinsam. Bei näherer Betrachtung erkennt das Fachauge jedoch einige Gemeinsamkeiten, die differentialdiagnostisch betrachtet nicht unerheblich sind: Neben der deutlichen Zunahme der Prävalenz für beide Erkrankungsgruppen in den letzten zwei Jahrzehnten liegt eine weitere Gemeinsamkeit in der Beteiligung eines fehlgesteuerten Immunsystems auf Grundlage einer multifaktoriellen Ätiologie. Die Hypersensitivität und die resultierenden Entzündungsprozesse werden entweder durch exogene oder endogene Antigene getriggert. Das Spektrum der immunologischen Antwort hinsichtlich zellulärer Differenzierung des adaptiven Immunsystems und beteiligter molekularer Mediatoren unterscheidet sich bei atopischen Erkrankungen zwar deutlich von dem der Autoimmunerkrankungen. Doch auch wenn die Atopie und die Autoimmunität somit als differente immunologische Zustände verstanden werden, lassen Ergebnisse aus diversen epidemiologischen Studien der letzten 10 Jahre überraschend viele Überschneidungen erkennen. Beispielsweise scheint die Atopie gerade in frühen Kinderjahren ein prädisponierender Faktor für autoimmune Schilddrüsenerkrankungen den juvenilen systemischen Lupus erythematodes wie auch für verschiedene Vaskulitiden $[3,4]$ darzustellen.

\section{Studienergebnisse}

Trotz aller Limitierungen, die der Natur retrospektiver Studien geschuldet sind, legen die Ergebnisse der vorliegenden israelischen Studie die Atopie als einen prädisponierenden Faktor für das insgesamt seltene Kawasaki-Syndrom nahe. Auf der pathophysiologischen Ebene konnten die Mechanismen, die einen Zusammenhang zwischen Autoimmunität und Atopie erklären könnten, bisher noch nicht hinreichend belegt werden. Als mögliche Bindeglieder werden Interaktionen dendritischer Zellen, regulatorischer T-Zellen, Th17-Zellen sowie Interleukin-9 diskutiert. Fortschritte in dieser Fragestellung könnten Implikationen für präventive therapeutische Maßnahmen bereithalten.

\section{Fallbeispiel aus der Praxis}

In unserer kinderpulmologischen Ambulanz des Universitäts Kinder- und Frauenzentrums in Dresden betreuen wir eine junge Pa- tientin mit den klassischen Manifestationen der Atopie: Neurodermitis, allergische Rhinokonjunktivitis und Asthma bronchiale. Das besondere bei diesem Mädchen waren im Verlauf zusätzlich auftretende Autoimmunphänome wie Alopecia totalis, Infektanfälligkeit und chronische Immunthrombozytopenie. Seither erfolgt eine interdisziplinäre Betreuung durch Immunologen, Humangenetiker und Hämatologen. Noch ist die Ätiologie nicht vollständig geklärt. Zudem drängt sich die Frage auf, ob in diesem Falle die Atopie als eine Koinzidenz oder gar als Prädisposition für die Autoimmunphänomene vorliegt.

\section{Fazit für die Praxis}

Für die Praxis und die Arbeit mit pädiatrischen Patienten mag es bisweilen hilfreich sein, die Atopie als prädisponierenden Faktor für Autoimmunerkrankungen in differentialdiagnostische Überlegungen einzubeziehen.

\section{Disclosure Statement}

Hiermit erkläre ich, dass ich keine Interessenskonflikte in Bezug auf den vorliegenden Wissenstransfer bestehen.

\section{Erstveröffentlichung}

Dieser Beitrag wurde erstveröffentlicht in: Karger Kompass Pneumol 2017;5:229-230.

\section{Literatur}

1 D'Angelo G, Marseglia L, Manti S, et al.: Atopy and autoimmune thyroid diseases: melatonin can be useful? Italian J Pediatr 2016;42:95.

2 Guo R, Zhou Y, Lu L, et al.: Atopy in children with juvenile systemic lupus erythematosus is associated with severe disease. PLoS One 2017;17: e0177774.

3 Wei C-C, Lin C-L, Shen T-C, et al.: Atopic dermatitis and association of risk for Henoch-Schönlein purpura (IgA vasculitis) and renal involvement among children: results from a population-based cohort study in Taiwan. Medicine (Baltimore) 2016;95:e2586.

4 Greco A, Rizzo MI, De Virgilio A, et al.: Churg-Strauss syndrome. Autoimmun Rev 2015;14:341-348

Kontaktadresse: David Brandt, Universitäts Kinder- und Frauenklinik, Klinik für Kinder- und Jugendmedizin, Bereich Bronchopneumologie/ Allergologie, Fetscherstraße 74, Haus 21, 01307 Dresden, Deutschland, david.brandt@uniklinikum-dresden.de 